\title{
Kontroversen in der systemischen Primärtherapie des Mammakarzinoms
}

\author{
C. Jackisch \\ Klinik und Poliklinik für Geburtshilfe und Frauenheilkunde der Westfälischen Wilhelms-Universität Münster
}

Das therapeutische Konzept des Mammakarzinoms beinhaltet neben operativen und strahlentherapeutischen Maßnahmen zur Optimierung der lokalen Tumorkontrolle, eine Vielzahl von systemischen Behandlungsmöglichkeiten, um das krankheitsfreie Intervall bzw. das Gesamtüberleben zu verbessern. Für das nodal-negative Mammakarzinom besteht dabei prinzipiell ein kurativer Therapieansatz. Die Validierung neuer Therapiekonzepte gegenüber dem derzeitigen klinischen Standard ist nur im Rahmen von klinischen Studien möglich. Bedauerlicherweise werden in Deutschland nur maximal 5\% aller Mammakarzinome in Studien behandelt. Diese neuen Erkenntnisse werden im Rahmen von internationalen Konsensus-Empfehlungen evaluiert und finden somit Eingang in neue Therapiekonzepte der klinischen Routine. Eine der weltweit bedeutendsten Konsensus-Konferenzen findet alle drei Jahre in St. Gallen statt - zuletzt 1998.

\section{Bedeutung der Dosisintensität}

Die Arbeitsgruppe um Bonadonna [1] konnte belegen, daß von einer adjuvanten Chemotherapie nach dem Cyclophosphamid, Methotrexat, 5-Fluorouracil (CMF)-Protokoll nach 20 Jahren insbesondere prämenopausale Frauen mit 1-3 positiven Lymphknoten im Hinblick auf das krankheitsfreie (disease free survival, DFS) sowie das Gesamtüberleben (overall survival, OAS) profitieren (DFS: 29 vs. 37\%, OAS: 24 vs. 38\%). Dieser Effekt konnte für die Gruppe der nodal-positiven Mammakarzinome ( $\geq 4$ Lymphknoten positiv) nicht gezeigt werden. Für die zytotoxische Systemtherapie ist die Einhaltung der geplanten Dosis von essentieller Bedeutung. Dosisreduktionen und Intervallverlängerungen bedingen eine Reduktion der Dosisintensität (DI in $\mathrm{mg} / \mathrm{m}^{2} /$ Woche) und gefährden den erwünschten Therapieerfolg (Abb. 1). Durch den Einsatz hämatopoetischer Wachstumsfaktoren (z. B. G-CSF) konnten hier erhebliche Verbesserungen etabliert werden.

\section{Primäre «neoadjuvante» Chemotherapie des operablen Mammakarzinoms}

Die systemische Primärtherapie des Mammakarzinoms hat durch die zunehmende Anwendung der primären «neoadju- vanten» Therapie des operablen Mammakarzinoms einen entscheidenden Wandel erfahren. Auch hier haben klinische Studien (u. a. NSABP B-18) gezeigt, daß durch die Umkehr der klassischen Sequenz $(\mathrm{OP} \rightarrow$ Chemotherapie vs. Chemotherapie $\rightarrow$ OP) gleiche Ergebnisse für das DFS bzw. OAS erreichbar sind. Neben der Steigerung der brusterhaltenden Primäroperationen zeigt sich für diejenigen mit einer pathologisch kompletten Remission (etwa 10\% pathologic complete remission (pCR)) ein Überlebensvorteil. In einer Pilotphase konnten von Minckwitz et al. [2] zeigen, daß 4 Zyklen einer intervallverkürzten, und somit dosisintensivierten, 14tägigen primären Chemotherapie (ADOC-Schema: Adriamycin $50 \mathrm{mg} / \mathrm{m}^{2}+$ Docetaxel $75 \mathrm{mg} / \mathrm{m}^{2}+$ G-CSF) sicher durchführbar sind und nach einer Therapiedauer von 8 Wochen ein Gesamtansprechen von $93 \%$ sowie eine sonographisch evaluierte CR von $67 \%$ erreicht werden konnte. Im Rahmen der am 14.06.1999 abgeschlossenen Phase-IIB-Studie (GEPARDO) wurden $255 \mathrm{~Pa}-$ tientinnen randomisiert nach dem ADOC-Schema \pm Tamoxifen $30 \mathrm{mg}$ behandelt. Hier konnte bisher eine brusterhaltende Therapierate von $71 \%$ erzielt werden. Die Nachfolge Phase-III-Studie GEPARDUO (Abb. 2, 3) prüft nun für primäre Mammakarzinome $\geq 2 \mathrm{~cm}$ die Kombination ADOC + Tamoxifen $30 \mathrm{mg}(\times 4 \mathrm{q} 14 \mathrm{~d})$ gegen den Sequenzarm der NSABP B-27 (Adriamycin / Cyclophosphamid (AC) $\times 4 q 21 d$ $\rightarrow$ Docetaxel $\times 4 q 21 d)$.

\section{Stand der konventionellen adjuvanten Chemotherapie des Mammakarzinoms}

Für die klinische Routine gelten derzeit die Empfehlungen der 1998 aktualisierten Konsensus Konferenz von St. Gallen [3]. Hiernach gilt, daß bis auf rezeptorpositive Karzinome $(\leq 1 \mathrm{~cm}+$ Grading I), für alle Mammakarzinome eine endokrine oder zytotoxische Therapie empfohlen wird. Die 1998 publizierte Metaanalyse der Early Breast Cancer Trialists' Collaborative Group (EBCTCG) [4] läßt erkennen, daß der Einsatz einer Chemotherapie im Hinblick auf DFS und OAS eindeutige Vorteile ergibt. Insgesamt wurden 47 Studien (18.000 Patienten nodal-negativ/positiv) analysiert. Der Stellenwert der Anthrazykline für nodal-positive Mammakarzinome wurde an 6.000 Patienten (11 Studien) überprüft. Im Hinblick auf die Mortali-

\begin{tabular}{ll}
\hline KARGER & @ 1999 S. Karger GmbH, Freiburg \\
Fax +49 7614520714 & Accessible online at: \\
$\begin{array}{l}\text { E-mail kargergmbh@aol.com } \\
\text { www.karger.com }\end{array}$ & http://BioMedNet.com/karger
\end{tabular}

Dr. med. Christian Jackisch

Klinik und Poliklinik für Geburtshilfe und Frauenheilkunde der Westfälischen Wilhelms-Universität Münster Albert-Schweitzer-Straße 33, D-48129 Münster (Deutschland) Tel./Fax 0251/834-8108

E-mail jackisch@uni-muenster.de 


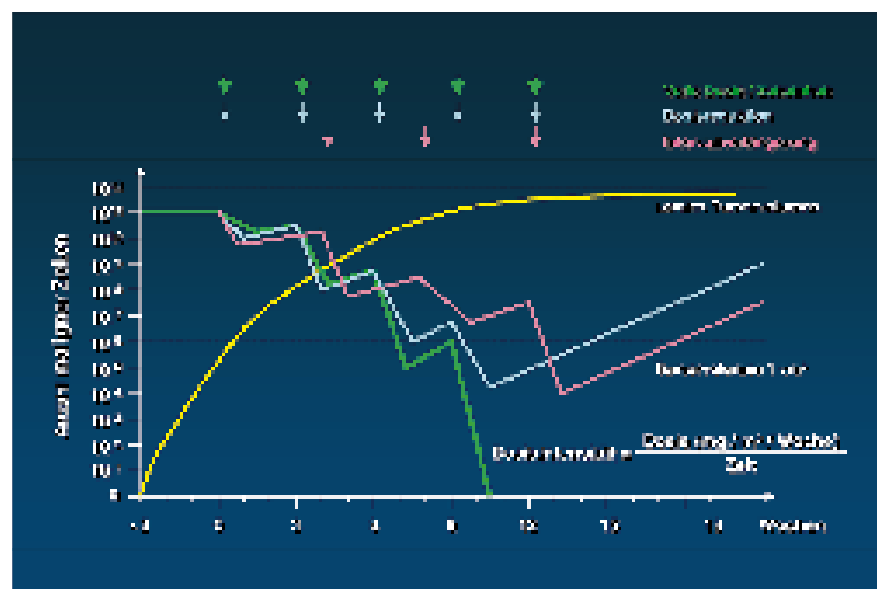

Abb. 1. Bedeutung der Dosisintensität. Dosisreduktionen und Intervallverlängerungen. Intervallverlängerungen führen zur Reduktion der geplanten Dosisintensität.

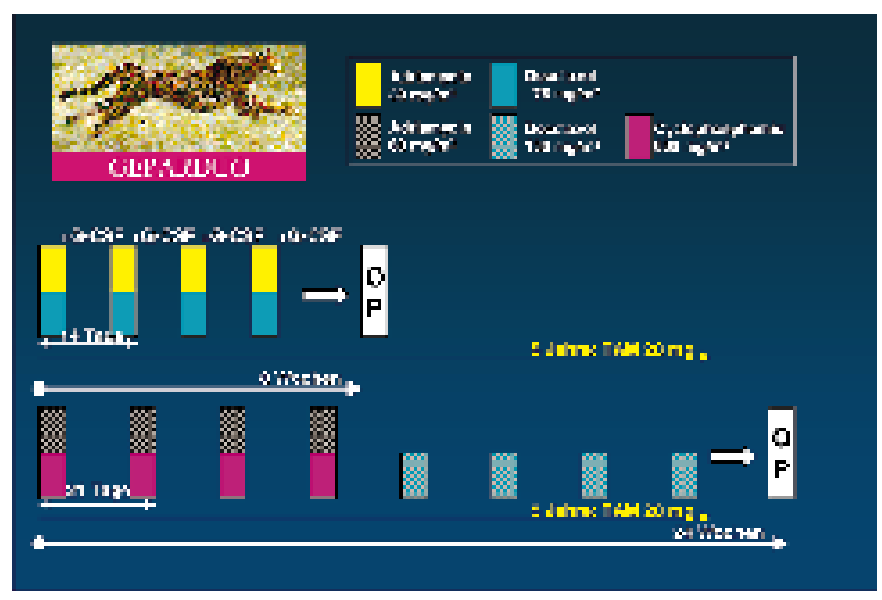

Abb. 2. GEPARDUO-Protokoll.

tätsreduktion besteht hier der größte Nutzen von $11 \%$ für das Kollektiv nodal-positiv $(\mathrm{N}+),<50$ Jahre, gefolgt von $7 \%$ für nodal-negativ ( $\mathrm{N}-),<50$ Jahre. Levine et al. [5] konnten im Vergleich Cyclophosphamid/Epirubicin/5-Fluorouracil (CEF) versus $\mathrm{CMF}$ bei prämenopausalen, nodal-positiven Frauen einen Vorteil des Anthrazyklin-haltigen Schemas von 63 versus 53\% (5-Jahres-DFS, $p=0,009$ ) und 77 versus 70\% (5-JahresOAS, $p=0,03)$ belegen. Für die Bedeutung der Einführung der Taxane in das adjuvante Therapiekonzept muß die 1998 publi-

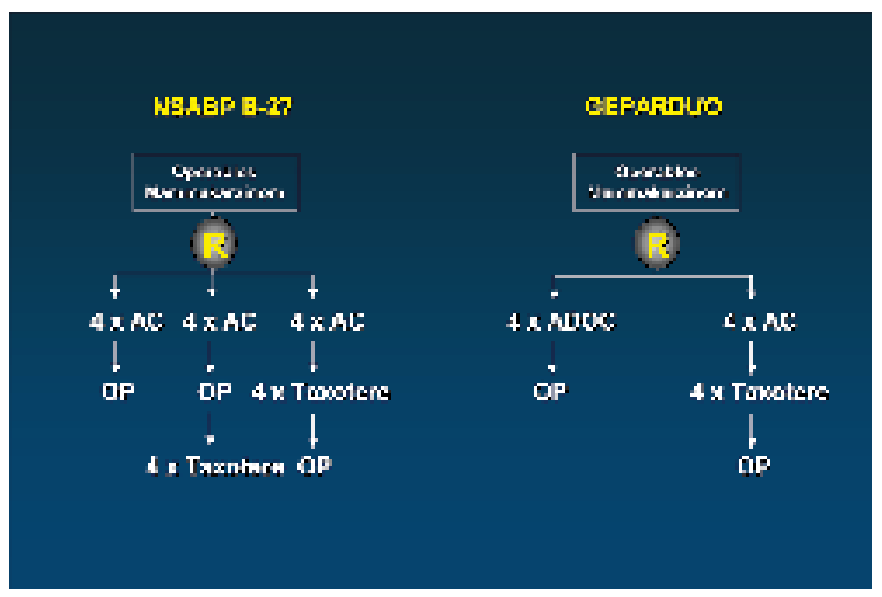

Abb. 3. GEPARDUO-Studie.

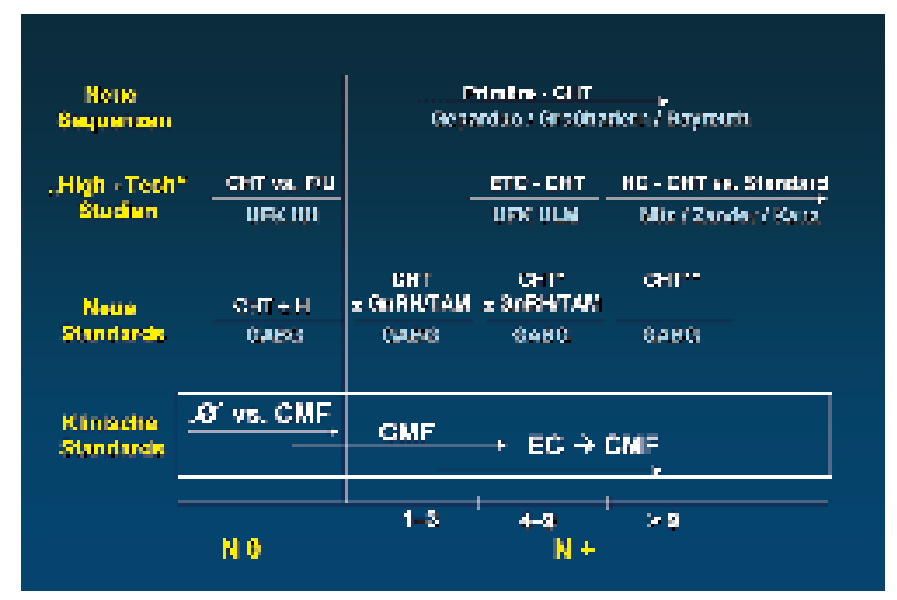

Abb. 4. Systemische Primärtherapie des Mammakarzinoms. Aktueller Stand in Deutschland unter Einschluß aktiver klinischer Studien. CHT = Chemotherapie, $\mathrm{F} / \mathrm{U}=$ Follow-up, UFK = Universitäts-Frauenklinik, $\mathrm{HH}=$ Hamburg, ETC = Epirubicin /Taxol/Cyclophosphamid, HD = HochdosisChemotherapie, TAM = Tamoxifen, $\mathrm{GABG}=$ German Adjuvant Breast Cancer Study Group, EC = Epirubicin/Cyclophosphamid.

zierte Studie von Henderson et al. [6] (AC vs. AC $\rightarrow$ Paclitaxel) beachtet werden. Hier zeigt sich bei einem Kollektiv von 3.170 Patienten ein signifikanter Vorteil für den sequentiellen Einsatz des Taxans (DFS: 90 vs. $86 \%, p=0,007$; OAS: 97 vs. $95 \%, \mathrm{p}=0,039)$. Die derzeitige Situation der adjuvanten systemischen Primärtherapie in Deutschland, unter Einschluß der aktiven klinischen Studien, ist abschließend graphisch dargestellt (Abb. 4). 


\section{Literatur}

1 Bonadonna G, Valagussa P, Moliterni A, Zambetti M: Adjuvant cyclophosphamide methotrexate and fluorouacil in node-positive Breast Cancer. N Engl J Med 1995;332:901-906.

2 von Minckwitz G, Costa SD, Eiermann W, Blohmer JU, Tulusan AH, Jackisch C, Kaufmann M: Maximized reduction of primary breast tumor size using preoperative chemotherapy with doxorubicin and docetaxel. J Clin Oncol 1999;17:1999-2005.

3 Goldhirsch A, Glick JH, Gelber RD, Senn HJ Meeting highlights: International consensus panel on the treatment of primary breast cancer. J Natl Cancer Inst 90;1998:1601-1608.
4 Clarke M, Collins R, Davies C, Godwin J, Gray R, Peto R: Polychemotherapy for early breast cancer: an overview of the randomized trials. Lancet 1998;352 930-942.

5 Levine MN, Bramwell VH, Pritchard KI, Norris BD, Shepard LE, Abu-Zahra H, Findlay B, Warr D Bowman D, Myles J, Arnold A, Vandenberg T, MacKenzie R, Robert J, Ottaway J, Burnell M, Williams CK, Dongsheng Tu, for the National cancer Institute of Canada Clinical Trialist Group: Randomized trial of intensive cyclophosphamide, epirubicin, and fluorouracil chemotherapy compared with cyclophosphamide, methotrexate, and fluorouracil in premenopausal women with node-positive breast cancer. J Clin Oncol 1998;16:2651-2658.
6 Henderson IC, Berry D, Demetri G, Cirrincione L, Goldstein L, Martino S, Ingle JN, Cooper MR, Cannelos G, Borden E, Femming G, Holland JF, Graziano S Carpenter J, Muss H, Norton L: Improved diseasefree (DFI) and overall survival (OAS) from the addition of sequential paclitaxel $(\mathrm{T})$ but not from the escalation of doxorubicin (A) dose level in the adjuvant chemotherapy of patients (PTS) with node-positive primary breast cancer. Proc Am Soc Clin Oncol 1998;17:\#390A. 